\title{
Spatiotemporal Formation Kinetics of Polyelectrolyte Complex Mi- celles with Millisecond Resolution
}

\author{
Hao Wu, ${ }^{\dagger, \#}$ Jeffrey M. Ting, ${ }^{\dagger,}, \#$ Boyuan Yu, ${ }^{\dagger}$ Nicholas E. Jackson, ${ }^{\dagger}, \star$ Siqi Meng, ${ }^{\dagger}$ Juan J. de Pablo, ${ }^{\dagger, \$}$ \\ and Matthew V. Tirrell ${ }^{\dagger}, * *$ \\ ${ }^{\dagger}$ Pritzker School of Molecular Engineering, University of Chicago, Chicago, Illinois 60637, United States. \\ $\star$ Center for Molecular Engineering and Materials Science Division, Argonne National Laboratory, Lemont, Illinois 60439, \\ United States.
}

\begin{abstract}
We have directly observed the in situ self-assembly kinetics of polyelectrolyte complex (PEC) micelles by synchrotron time-resolved small-angle X-ray scattering, equipped with a stopped-flow device that provides millisecond temporal resolution. A synthesized neutral-charged diblock polycation and homopolyanion that we have previously investigated as a model charge-matched, core-shell micelle system were selected for this work. The initial micellization of the oppositely charged polyelectrolytes was completed within the dead time of mixing of $100 \mathrm{~ms}$, followed by micelle growth and equilibration up to several seconds. By combining the structural evolution of the radius of gyration $\left(R_{\mathrm{g}}\right)$ and aggregation number $(N)$ with complementary molecular dynamics simulations, we develop new information on how the self-assemblies evolve incrementally in size over time through a two-step kinetic process: first, oppositely-charged polyelectrolyte chains pair to form nascent aggregates that immediately assemble into spherical micelles, and second, these PEC micelles grow into larger micellar entities. This work has determined one possible kinetic pathway for the initial formation of PEC micelles, which provides useful physical insights for increasing fundamental understanding selfassembly dynamics driven by polyelectrolyte complexation that occur on ultrafast timescales.
\end{abstract}

Under selective solution conditions, amphiphilic block polymers can be designed to self-assemble into an assortment of diverse morphological structures, depending on the relative length, chemical nature, and molecular architecture of the hydrophilic and hydrophobic polymer blocks. ${ }^{1,2}$ Equilibrium structures have received far more attention than the time-dependent phenomena of their formation. Theoretical works by Aniansson and Wall, ${ }^{3-5}$ Halperin and Alexander, ${ }^{6}$ and Dormidontova $^{7,8}$ have proposed two possible mechanisms for the kinetics of polymeric self-assemblies toward equilibrium: $(i)$ single-chain insertion/expulsion and (ii) micelle fusion/fission. Experimental evidence has tested these model frameworks by adjusting solvent quality to induce micelle formation. For instance, using millisecond time-resolved small-angle X-ray scattering (TR-SAXS) under stopped-flow to provide nanoscale spatial resolution and temporal resolution, Lund and coworkers reported that the initial micellization kinetics of poly(ethylenealt-propylene)-block-poly(ethylene oxide) (PEP- $b$-PEO) can be viewed as a fast nucleation (within $10 \mathrm{~ms}$ ) and a slow growth process, where the elemental PEP- $b$-PEO aggregate growth follows a single-chain insertion mechanism akin to the AnianssonWall theory. ${ }^{9}$ By comparison, Kalkowski et al. investigated micellization kinetics of poly(ethylene glycol)-block-poly(caprolactone) (PEG- $b$-PCL) with synchrotron X-ray scattering equipped with a microfluidic device. ${ }^{10}$ In this system, they distinguished three successive stages of the micellization (nucleation, micelle fusion, and polymer insertion), evident by the temporal evolution of the radii of gyration $\left(R_{\mathrm{g}}\right)$. From $0-250 \mathrm{~ms}$, the $R_{\mathrm{g}}$ increased steadily as free unimers coupled into nuclei. Immediately after $250 \mathrm{~ms}$, the $R_{\mathrm{g}}$ abruptly doubled in size, indicating that nascent nuclei began merging together, followed by steady structural growth from free PEG- $b$-PCL chains. While distinct from the Halperin-Alexander and Lund reports, the formation kinetics are in good agreement with the particle-particle fusion model described by Dormidontova. ${ }^{8}$ Further advanced techniques such as in situ liquid-cell transmission electron microscopy and in silico simulations have captured such collision events in solution. ${ }^{11}$ In short, understanding kinetic formation in terms of tunable fundamental variables (e.g., polymer size/composition, concentration, interfacial tension) has unlocked design principles to more easily prepare nanoparticle assemblies with greater fidelity, control, reproducibility, and stability over time for intended applications.

However, compared to the advances in understanding the assembly kinetics of micelles formed by uncharged amphiphilic polymers over the past decade, ${ }^{6,8,12-18}$ little is known about the initial moments of self-assembly of polyelectrolyte complexation-driven systems. Polyelectrolyte complexes (PECs) are prepared from an associative phase separation (i.e., coacervation) process between oppositely charged polyelectrolytes in water; for block polyelectrolytes, the process is thought to be more complicated due to the interplay among multiple components, including the polycation/polyanion pairing (e.g., polymer polarity, charge density, sequence effect, chirality, asymmetric block length ratios, etc.), high water content and related excluded volume considerations, and the presence of salts in physiological settings. ${ }^{19-21}$ Since the formation of PEC micelles is believed to be strongly driven by the entropically favorable liberation of counterions between oppositely charged moieties, it is reasonable to infer that there are parallels to the thermodynamics of homopolyelectrolyte complexation. ${ }^{22,23}$ Takahashi and coworkers 
investigated the complexation kinetics of oppositely charged homopolyelectrolytes, sodium poly(acrylate) and poly(allylamine hydrochloride), in aqueous $\mathrm{NaCl}$ solutions with TR-SAXS coupled with a stopped-flow device. ${ }^{24}$ They reported the evolution of the complex droplet size, structure, and molar mass in a time range from 2.5 to $8733 \mathrm{~ms}$, following $(i)$ an immediate (< $2.5 \mathrm{~ms}$ ) pairing step where oppositely charged chains pair by electrostatic interactions; (ii) a complexation step where the ion pairs further coalesce into nearly neutral aggregates, driven by van der Waals and hydrophobic effects; (iii) a growth step where aggregates grew analogously to the Brownian-coagulation kinetics of spherical colloidal particles. The same group also later revealed morphological transitions of cylindrical-tospherical PEC micelles with salt by TR-SAXS, ${ }^{25}$ similarly to amphiphilic micelles. ${ }^{26}$

In general, the self-assembly process of PEC micelles from well-defined polyelectrolyte systems has remained unclear. In this Letter, by employing in situ TR-SAXS with millisecond temporal resolution, we experimentally investigate the self-assembly of one set of well-defined PEC micelles as a starting point toward building more quantitative predictions of formation kinetics. The constituents of this model PEC micelle system are poly(ethylene oxide)-block-poly(vinyl benzyl trimethylammonium chloride) (PEO- $b$-PVBTMA) and sodium poly(acrylate) (PAA), shown in Figure 1-A. We have previously demonstrated the controlled fabrication of polyelectrolytes in parallel synthesis ${ }^{27}$ and interrogated aspects of the welldefined equilibrium structures of this pairing in the dilute regime. ${ }^{28,29}$ TR-SAXS experiments were carried out on the Biological Small Angle Scattering Beamline BL 4-2 at the Stanford Synchrotron Radiation Lighthouse (SSRL), SLAC National Accelerator Laboratory. ${ }^{30,31}$ The TR-SAXS facility at the SSRL BL4-2, equipped with a customized Bio-Logic 4 syringe stopped-flow mixer (SFM 400), provides access to solution kinetics on the millisecond time scale. Briefly, a typical TRSAXS experiment proceeds in the following manner, shown schematically in Figure 1-B. First, aqueous solutions of positively-charged polymers and negatively-charged polymers were loaded in two separate syringes. Second, equal volumes of polycation and polyanion solutions were pumped into the mixer by the motors, corresponding to the equimolar concentration of the cationic and anionic monomer units. The dead time was $\sim 4 \mathrm{~ms}$. Third, $30 \mu \mathrm{L}$ of mixed solution was further dispensed into the capillary cell, where the incident X-ray radiates through. The sample solution was sealed and buffered by solvent on the two ends of the capillary cell. The exposure time for each measurement was set to $20 \mathrm{~ms}$. Automated capillary cleaning assisted in checking for potential radiation damage between experiments. Additional details are provided in the Supporting Information.

The time evolution of the SAXS curves is presented in Figure 2. Three independent kinetic experiments were carried out from the 5K53-158 sample, where the diblock polycation was $\mathrm{PEO}_{5 \mathrm{~K}}-b-\mathrm{PVBTMA}_{53}$ (molar mass of PEO $=5000 \mathrm{~g} \mathrm{~mol}^{-1}$; degree of polymerization of PVBTMA $=53$ ), the polyanion was $\mathrm{PAA}_{158}$ (degree of polymerization of PAA $=158$ ), and the total polymer concentration was $2.5 \mathrm{mg} \mathrm{mL}^{-1}$. To achieve high time resolution, we used a low exposure time of $20 \mathrm{~ms}$, which may reduce the quality of the scattering curves due to noise. To avoid this, we independently repeated the same experiment three times. It is shown that the scattering data are reliable and reproducible even under a very low exposure time of $20 \mathrm{~ms}$. Collectively, all TR-SAXS patterns exhibited features of isolated particles with spherical morphology, well fitted by a polydisperse core-shell sphere model (see the Supporting Information). The increase in the scattering intensity over time reflects the micelle growth captured in real time. Due to the limitations of the instrument setup and experimental conditions, the lower limit of the experimentally probed time period remained $100 \mathrm{~ms}$. Thus, the nucleation event for chain complexation was completed by $80 \mathrm{~ms}$ and was not experimentally observable.

A

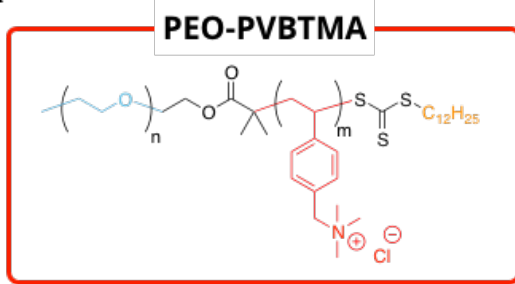

$5 K 50$
$10 \mathrm{~K} 100$

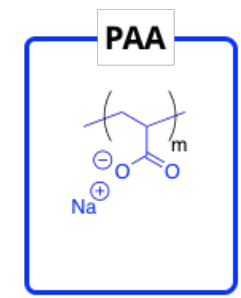

158
B

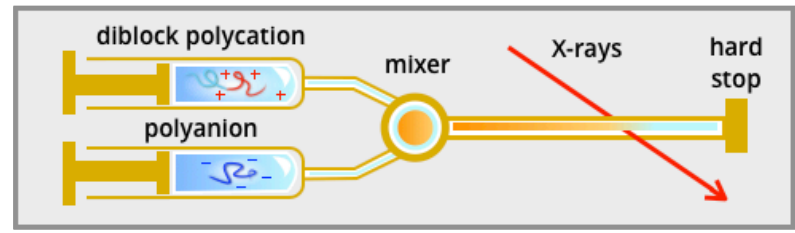

Figure 1. (A) Polyelectrolyte complex micelles consist of a polycation, PEO- $b$-PVBTMA, and polyanion, PAA. (B) Schematic representation of the time-resolved small-angle X-ray scattering experiments equipped with a stopped-flow setup.

Nevertheless, the structural evolution of the 5K53-158 PEC micelles provided insightful information in the time range from 100 to $5000 \mathrm{~ms}$. At $100 \mathrm{~ms}$, well-formed micellar aggregates with a spherical geometry were already present in solution. The scattering intensity values increased steadily over time while maintaining the overall shape until $5000 \mathrm{~ms}$, when the change in scattering intensity was barely discernable and indicated that the micelle growth became exceptionally slow relative to the prior state. The final state of micelles formed after injection in the stopped-flow path was sampled with SAXS after several minutes and did not differ (see Supporting Information). We also investigated the effects of block length and polymer concentration on the formation kinetics of PEC micelles, particularly on the growth rate. This involved a longer polycation, PEO $_{10 \mathrm{~K}}-b$-PVBTMA 100 with PAA $_{158}(10 \mathrm{~K} 100-158)$ at 2.5 and $3.75 \mathrm{mg} \mathrm{mL}^{-1}$. In anticipation of slower growth kinetics, the exposure time was set to $80 \mathrm{~ms}$ in order to extend experimental time window for TR-SAXS characterization. The recorded time was $19,200 \mathrm{~s}$ and $38,400 \mathrm{~s}$ for the 2.5 and $3.75 \mathrm{mg} \mathrm{mL}^{-1}$ polymer concentrations, respectively. We observed that qualitatively, 10K100-158 samples formed larger micellar aggregates at a slower overall rate at both concentrations. 

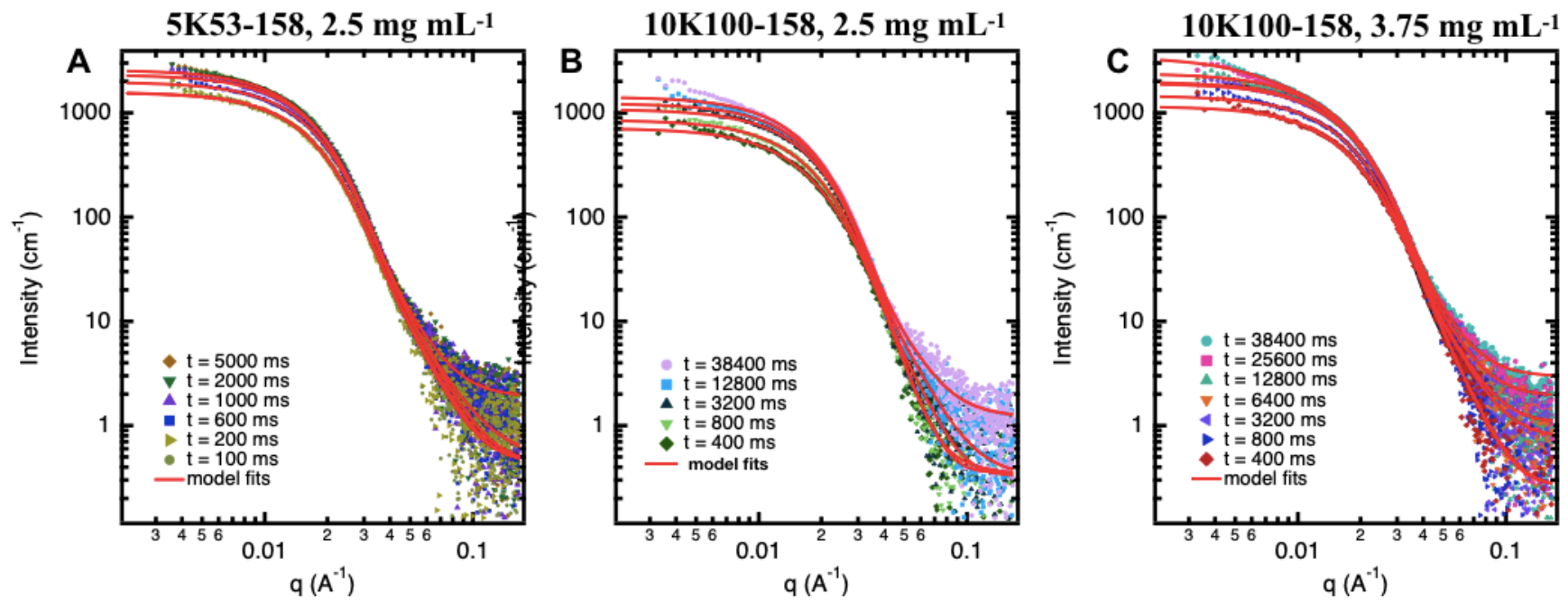

Figure 2. TR-SAXS curves showing the formation of PEC micelles by (A) $5 \mathrm{~K} 53-158$ at $2.5 \mathrm{mg} \mathrm{mL}^{-1}$ total polymer concentration, and (B) $10 \mathrm{~K} 100-158$ at 2.5 and (C) $3.75 \mathrm{mg} \mathrm{mL}^{-1}$ total polymer concentration, respectively. Data points represent experimental data; see Figure S-X for fittings to a polydisperse core-shell model.

To better understand the assembly mechanism, the micelle size evolution over time was quantified. The $R_{\mathrm{g}}$ of the micellar aggregates over time was extracted via the Guinier approximation using the GNOM package ${ }^{32}$ as we have previously described $^{28}$ The aggregation number $(N)$ was determined from fitting to a polydisperse core-shell sphere model. Three independent measurements of 5K53-158 formation showed a similar time dependence. As seen in Figure 3, we observe that at within the initial $100 \mathrm{~ms}$, PEC micelles have already formed with $R_{\mathrm{g}}=$ $10.5 \mathrm{~nm}$. Afterward, these micellar aggregates continued to grow in size, steadily increasing by $14 \%$ in $R_{\mathrm{g}}$ over $5000 \mathrm{~ms}$. By dynamic light scattering (see Supporting Information), preassembled micelles prepared from these polyelectrolytes generally exhibit an apparent hydrodynamic radius $\left(R_{\mathrm{h}}\right)$ of ca. 30.0 $\mathrm{nm}$, corresponding to a shape factor $\left(R_{\mathrm{g}} / R_{\mathrm{h}}\right)$ of 0.4 . This corresponds to a highly water solvated structure, well below the conventional reference of a solid sphere $\left(R_{\mathrm{g}} / R_{\mathrm{h}}=\sqrt{3 / 5} \sim 0.77\right){ }^{33}$ For comparison, Heo et al. have reported molecular conformation measurements of multiple post-polymerization functionalized PEC micelles, which comprised roughly $80 \%$ water by neutron scattering. ${ }^{34} \mathrm{~A}$ similar trend in $R_{\mathrm{g}}$ and $N$ versus time was found in the $10 \mathrm{~K} 100-158$ samples in Figure 4 . The kinetic equilibration process appears slower for the micelles with longer polycation block lengths. For the 5K53-158 micelle, the $R_{\mathrm{g}}$ values plateaued around $5000 \mathrm{~ms}$, whereas for the $10 \mathrm{~K} 100-$ 158 system, the $R_{\mathrm{g}}$ increased by $27 \%$ from $11.0 \mathrm{~nm}$ at $5000 \mathrm{~ms}$ to $14.0 \mathrm{~nm}$ at $38,400 \mathrm{~ms}$. We attribute this delay to the higher activation energy barrier and longer time involved with polymer rearrangement in longer chains.

From the aforementioned amphiphilic block polymer literature, there are two conventional mechanisms for micelle formation: single-chain insertion/expulsion or micelle fusion/fission. Micelles formed by oppositely charged polymers have features that are distinct from uncharged polymers. According to the current theories of polyelectrolyte complexation, free individual chains are driven to form complexes with their oppositely charged counterparts due to predominately entropic contributions, ${ }^{35-37}$ even at extremely low concentrations. Under this established framework, free chains are unlikely to exist as unbound chains when micelles have already formed. In alignment with time-resolved formation kinetics using homopolyelectrolytes, ${ }^{38}$ it is reasonable to infer that a similar entropically-driven process occurs with block polyelectrolytes: immediately upon mixing, oppositely charged blocks pair with each other to form effectively neutralized clusters within a few milliseconds. Chain exchange events may happen immediately after complexation, but given the short time and length scales, this is not experimentally observable, even with advanced synchrotron capabilities. However, in the hundred-millisecond time periods, the experimental data suggests that it is unlikely that micelle fusion/fission took place. Otherwise, the $R_{\mathrm{g}}$ values of the micellar aggregates would have exhibited an abrupt jump in magnitude, as Kalkowski et al. have observed in the (1) nucleation, (2) fusion, and (3) insertion stages of PEG- $b$-PCL micelle formation; here, the Guinier $R_{\mathrm{g}}$ correspondingly evolved from (1) $\sim 2-5 \mathrm{~nm}$ to (2) $\sim 5-9 \mathrm{~nm}$, before (3) plateauing at $\sim 10 \mathrm{~nm} .{ }^{10}$

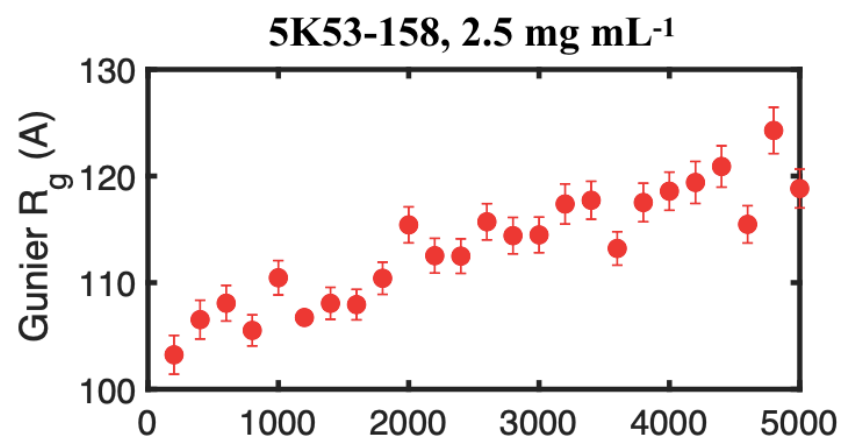

Figure 3. The evolution of the fitted Guinier $R_{\mathrm{g}}$ for $5 \mathrm{~K} 53-158$ (red circles) in TR-SAXS experiments over the time range of 0 to 5000 $\mathrm{ms}$. Error bars denote the standard deviation of each measurement. 

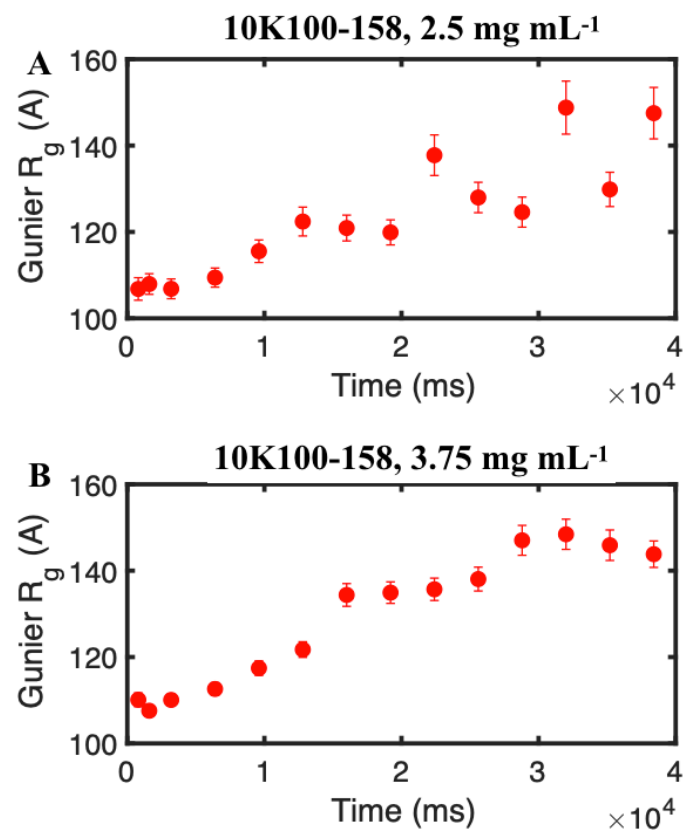

Figure 4. The evolution of the fitted Guinier $R_{\mathrm{g}}$ for $10 \mathrm{~K} 100-158$ (red circles) at (A) $2.5 \mathrm{mg} \mathrm{mL}^{-1}$ and (B) $3.75 \mathrm{mg} \mathrm{mL}^{-1}$ over the time range of 0 to $40,000 \mathrm{~ms}$. Error bars denote the standard deviation of each measurement. The final $R_{\mathrm{g}}$ and $N$ are $\mathrm{X}$ and $\mathrm{X}$, respectively.

Furthermore, when we repeated TR-SAXS measurements at a higher $3.75 \mathrm{mg} \mathrm{mL}^{-1}$ total polymer concentration, the slow growth of $R_{\mathrm{g}}$ remained invariant over time (Figure 4B). This implies that the formation of dilute PEO- $b$-PVBTMA/PAA micelles is largely concentration-independent. Previous work on electrostatic assemblies have shown a strong dependence on polymer concentration due to fusion-fission mechanisms. ${ }^{39}$

Because the PEC micelles were highly solvated and completely hydrophilic, this result was initially puzzling. However, we have previously shown that this PEO- $b$-PVBTMA/PAA system exhibits repulsive interparticle effects at dilute conditions using SAXS. ${ }^{28}$ Physically, we demonstrated that when two micelles approach, the PEO coronas may be compressed to some extent, but the depth of the compressed area is only half of the corona thickness. In other words, the micelle cores were unable to reach each other, which supports the observations that PEC micelle fusion/fission is improbable during the formation process. Similar steric arguments have been made by theories for certain amphiphilic block polymer micelles that do not exhibit fusion-based growth mechanisms. ${ }^{6}$ At some critical stage the cluster insertion becomes dominant when micelle coronas are too large and dense to be penetrated.

To complement the experimental TR-SAXS kinetics conditions and assess the relationship between $R_{\mathrm{g}}$ and $N$ in this time regime, a charged Kremer-Grest model ${ }^{40}$ was employed for the coarse-grained molecular dynamics (MD) simulation of polyelectrolyte coacervates. Polymers were simulated as beadspring chains. For simplicity in maintaining electroneutrality, the $5 \mathrm{~K} 53$ chain was simulated as a diblock with 110 and 50 monomer beads for the neutral and positively charged blocks, respectively; the homopolyanion comprised 150 negativelycharged monomer beads. Each bead was identical in size and interacted via the shifted and truncated Lennard-Jones potential. The connectivity of polymer beads was maintained by the finitely extensible nonlinear elastic (FENE) potential. The salt- free solvent was treated implicitly with a dielectric constant $\varepsilon=$ 78.5, and counterions were neglected. Spherical micelles at given aggregation numbers were pre-assembled and placed at the center of the simulation box. Pre-assembly was achieved by first uniformly placing the polymer chains on a spherical surface of given radius with the charged blocks pointing towards the center of the sphere. An energy minimization of the system was then performed using a conjugated gradient algorithm implemented in a large-scale atomic/molecular massively parallel simulator (LAMMPS ${ }^{41}$ ) with $10^{6}$ MD steps for equilibration. Afterward, a production run of $10^{6}$ timesteps was used to collect data every $10^{4}$ timesteps to calculate the $R_{\mathrm{g}}$, radial density profile, and radial distribution function of the micelles. A detailed simulation methodology is provided in the Supporting Information.

The MD simulation results in Figure 5 show how the $R_{\mathrm{g}}$ and asphericity parameter $\left(\mathrm{k}^{2}\right)$ of the PEC micelle correlates to the change in specified $N$. Given three selected aggregation numbers $\left(N=75,105\right.$, and 135), it is observed that the $R_{g}$ increased from $8.25 \pm 0.98 \mathrm{~nm}$ to $9.66 \pm 0.88 \mathrm{~nm}$ (i.e., a $17 \%$ difference), which is qualitatively consistent with experimental estimates of a $14 \%$ increase in $R_{\mathrm{g}}$ growth. Differences in the absolute $R_{\mathrm{g}}$ values from experiment can be attributed to the coarse-grained and implicit solvent nature of the simulations. Near zero values of the asphericity parameter (Figure 5) combined with the computed radial distribution functions (Figure S- $\underline{5}$ ) indicated that at all molecular weights micelles exhibit spherical morphologies with similar internal structures. Further computational efforts can help capture the initial micellization kinetics that are not experimentally observable. For instance, Bos and Sprakel recently employed Langevin dynamics simulations to study fast exchange dynamics. ${ }^{42}$ In the early simulation time steps of the micellization stage, they found that initially, polyelectrolyte chains adopted a relatively stretched configuration before rapidly forming aggregates by both expulsion/insertion and fusion/fission events, revealed by the decrease of size and release of counterions. Fusion and fission events were defined by the expulsion of small neutralized clusters containing a few polyelectrolytes from one micelle into another micelle.

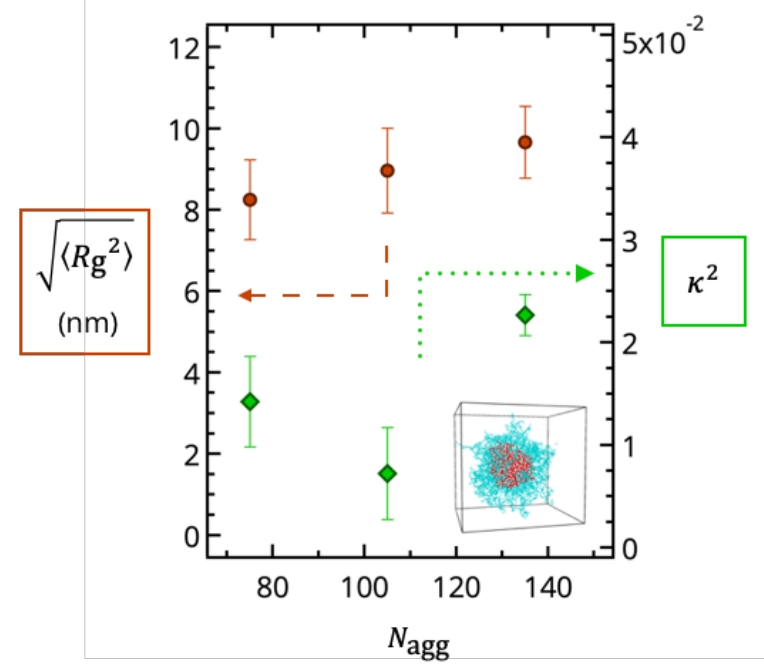

Figure 5. MD simulation results of the $R_{\mathrm{g}}$ (orange data with dashed arrow to the left axis) and asphericity (green data with dotted arrow to the right axis) as a function of aggregation number for the $5 \mathrm{~K} 53$ 158 system. The inset shows a snapshot of the micelle using LAMMPS analysis. 
Combining the experimental and simulation results with literature evidence, we propose that a possible kinetic pathway of micellization can be described in a two-stage process, as illustrated in Scheme 1. First, immediately upon mixing in the stopped-flow apparatus, oppositely charged polyelectrolytes form charge-neutralized clusters. From these cluster aggregates, although the ultrafast time scale of the PEC micellization process was not commensurate with TR-SAXS, well-defined spherical micelles with $R_{\mathrm{g}} \sim 10-11 \mathrm{~nm}$. Second, the micelle products equilibrate by exchanging either unimer chains or ionpaired clusters, as evident by incremental growth in $R_{\mathrm{g}}$ and $N$. During this process, we believe that fusion events are highly unlikely due to repulsive interparticle interactions that this system has been shown to exhibit. The universality of this kinetic pathway is the subject of ongoing investigations. In the former step, the mixing method of the stopped-flow apparatus and the ionic strength of the solution may play key roles in adjusting the timescale of cluster formation proceeding the PEO- $b$-PVBTMA and PAA micellization. For the latter step, greater scrutinization of the polyelectrolytes' physical attributes, e.g., varying the ratio and the total chain lengths of blocks, may reveal differences in the likelihood of micelle fusion/fission, and therefore, alter the spatiotemporal evolution of micelles upon equilibration.

Scheme 1. Illustrated kinetic pathways of the investigated PEC micelles.

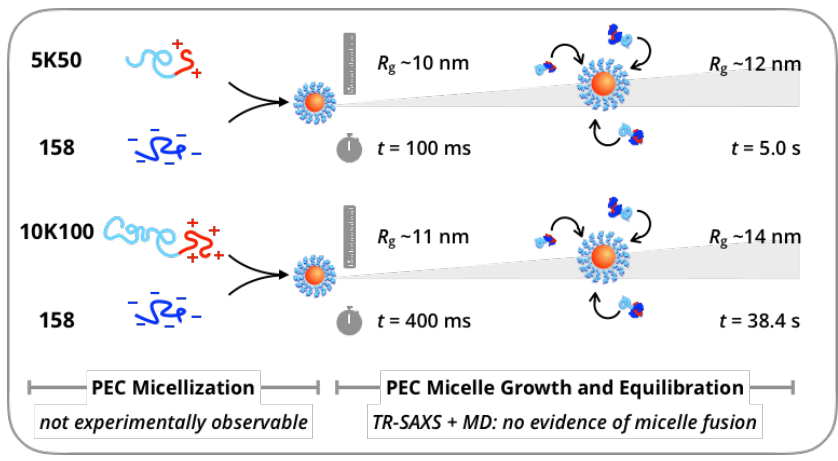

Furthermore, the formation kinetics may be strongly dependent on the chemical nature of the polymer constituents themselves. Charged heteropolymers and sequence-defined macromolecules give rise to distinct hierarchical assemblies at equilibrium, ${ }^{43}$ depending on the local interactions and sequence variation of the primary structure. By extension, the dynamics of PEC micelle formation, chain exchange, and equilibration are also likely highly influenced by how oppositely-charged polyelectrolytes associate at the molecular level. With this vast parameter space, greater systematic exploration of different polyelectrolytes is vital for more quantitative prediction capabilities and theories of complex coacervation. Recently, Amann et al. reported kinetic pathways for PEC micelle formation using TRSAXS using PEO- $b$-PVBTMA (2K6, 2K8, 2K12, and 2K19) and poly(styrene sulfonate) $\left(\mathrm{PSS}_{19}\right) .{ }^{44}$ Their data shows the formation of metastable aggregates, followed by rearrangement and pinch-off into smaller micellar-like entities. We hypothesize that this additional kinetic step arises because of the choice of the strongly-charged PSS polyanion. We have previously shown that upon complexation, PEO- $b$-PVBTMA forms micron-scale, kinetically frozen aggregates with PSS, in contrast to the spherical micelles with PAA. ${ }^{45}$ Furthermore, direct comparison of the homopolymer PVBTMA with PAA and PSS reveal liquid and solid complexes, respectively, by optical microscopy and SAXS (Figure S-1), which may account for subtle differences in the initial pairing step. Amann and coworkers also provide evidence that during the growth and equilibration step, fusion/fission events were not observed, in agreement with the subsequent behavior of PEO- $b$-PVBTMA/PAA. A direct comparison of formation pathways suggests that the early-stage micellization kinetics might be challenging to fully generalize, with the exact pathway likely to be highly dependent on the environmental and polymer parameters.

Altogether, this Letter provides an early study in understanding the ultrafast kinetics of ionic polymer assemblies. Further effort is underway to compare the kinetic pathway dependency of molecular attributes that are known to affect complex coacervation, such as the neutral-to-charged block lengths, the strength and type of the charged moieties, counterion valency, solvent quality, and other orthogonal association effects. Uncovering the underlying polymer physics from a fundamental molecular engineering standpoint can lead to new insights in an array of bioapplications. Such endeavors can potentially improve the loading capacity, robustness, and stability of non-viral vector formulations for proteins,${ }^{46}$ peptides,${ }^{47}$ and nucleic acids $^{48}$ in the future.

\section{ASSOCIATED CONTENT}

\section{Supporting Information}

The Supporting Information is available free of charge on the ACS Publications website.

Materials, Methods (file type, i.e., PDF)

\section{AUTHOR INFORMATION}

\section{Corresponding Author}

* E-mail: mtirrell@uchicago.edu (M.V.T.)

\section{ORCID}

Hao Wu: 0000-0001-6276-6114

Jeffrey M. Ting: 0000-0001-7816-3326

Boyuan Yu: 0000-0002-8200-4413

Nicholas E. Jackson: 0000-0002-1470-1903

Siqi Meng: 0000-0002-2238-9278

Juan J. de Pablo: 0000-0002-3526-516X

Matthew V. Tirrell: 0000-0001-6185-119X

\section{Notes}

The authors declare no competing financial interest. \#These authors contributed equally to this work.

\section{Author Contributions}

All authors contributed to proofreading the manuscript. H.W. conceived and implemented the project, carried out the micelle formation and scattering experiments, drafted the initial manuscript, and analyzed the scattering data. J.M.T. performed the polymer synthesis, carried out polymer characterization, and conducted the scattering experiments. B.Y. conducted the molecular dynamics simulations and analysis. N.E.J. assisted with the development and interpretation of the simulations. S.M. assisted with the scattering experiments and analyzed the bulk complex materials. J.J.d.P. was the principle investigator and contributed to the data analysis and interpretation. M.V.T. was the principal investigator of the project and directed the research, managed the project, and contributed to the data analysis and interpretation of the experiments. 


\section{Funding Sources}

This work was performed under the following financial assistant award 70NANB19H005 from U.S. Department of Commerce, National Institute of Standards and Technology (NIST) as part of the Center for Hierarchical Materials Design (CHiMaD).

\section{ACKNOWLEDGMENT}

We gratefully thank Artem M. Rumyantsev, $\mathrm{PhD}$, for helpful discussions. J.M.T. acknowledges support from the NIST-CHiMaD Postdoctoral Fellowship. Parts of this work were carried out at the Soft Matter Characterization Facility of the University of Chicago. The authors also thank Thomas Weiss, PhD, Ivan Rajkovic, PhD and Tsutomu Matsui, $\mathrm{PhD}$, at the Stanford National Accelerator Laboratory for their assistance in scattering experiments and insightful discussions. Use of the Stanford Synchrotron Radiation Lightsource, SLAC National Accelerator Laboratory, is supported by the U.S. Department of Energy, Office of Science, Office of Basic Energy Sciences under Contract No. DE-AC02-76SF00515. The SSRL Structural Molecular Biology Program is supported by the DOE Office of Biological and Environmental Research, and by the National Institutes of Health, National Institute of General Medical Sciences (including P41GM103393). The contents of this publication are solely the responsibility of the authors and do not necessarily represent the official views of NIGMS or NIH.

\section{REFERENCES}

(1) Cameron, N. S.; Corbierre, M. K.; Eisenberg, A. 1998 E.W.R. Steacie Award Lecture Asymmetric Amphiphilic Block Copolymers in Solution: a Morphological Wonderland. Can. J. Chem. 1999, 77 (8), 1311-1326.

(2) Moughton, A. O.; Hillmyer, M. A.; Lodge, T. P. Multicompartment Block Polymer Micelles. Macromolecules 2011, 45 (1), 2-19.

(3) Aniansson, E. A. G.; Wall, S. N. Kinetics of Step-Wise Micelle Association. J. Phys. Chem. 1974, 78 (10), 1024-1030.

(4) Aniansson, E. A. G.; Wall, S. N. Kinetics of Step-Wise Micelle Association. Correction and Improvement. J. Phys. Chem. 1975, $79(8), 857-858$.

(5) Aniansson, E. A. G.; Wall, S. N.; Almgren, M.; Hoffmann, H.; Kielmann, I.; Ulbricht, W.; Zana, R.; Lang, J.; Tondre, C. Theory of the Kinetics of Micellar Equilibria and Quantitative Interpretation of Chemical Relaxation Studies of Micellar Solutions of Ionic Surfactants. J. Phys. Chem. 1976, 80 (9), 905-922.

(6) Halperin, A.; Alexander, S. Polymeric Micelles: Their Relaxation Kinetics. Macromolecules 1989, 22 (5), 2403-2412.

(7) Esselink, F. J.; Dormidontova, E.; Hadziioannou, G. Evolution of Block Copolymer Micellar Size and Structure Evidenced with Cryo Electron Microscopy. Macromolecules 1998, 31 (9), 2925-2932.

(8) Dormidontova, E. E. Micellization Kinetics in Block Copolymer Solutions: Scaling Model. Macromolecules 1999, 32 (22), 76307644.

(9) Lund, R.; Willner, L.; Monkenbusch, M.; Panine, P.; Narayanan, T.; Colmenero, J.; Richter, D. Structural Observation and Kinetic Pathway in the Formation of Polymeric Micelles. Phys. Rev. Lett. 2009, 102 (18), 188301-188304.

(10) Kalkowski, J.; Liu, C.; Leon-Plata, P.; Szymusiak, M.; Zhang, P.; Irving, T.; Shang, W.; Bilsel, O.; Liu, Y. In Situ Measurements of Polymer Micellization Kinetics with Millisecond Temporal Resolution. Macromolecules 2019, 52, 3151-3157.

(11) Parent, L. R.; Bakalis, E.; Ramírez-Hernández, A.; Kammeyer, J. K.; Park, C.; de Pablo, J.; Zerbetto, F.; Patterson, J. P.; Gianneschi, N. C. Directly Observing Micelle Fusion and Growth in Solution by Liquid-Cell Transmission Electron Microscopy. J. Am. Chem. Soc. 2017, 139 (47), 17140-17151.
(12) Li, Z.; Dormidontova, E. E. Equilibrium Chain Exchange Kinetics in Block Copolymer Micelle Solutions by Dissipative Particle Dynamics Simulations. Soft Matter 2011, 7 (9), 4179-4188.

(13) Burke, S. E.; Eisenberg, A. Kinetics and Mechanisms of the Sphere-to-Rod and Rod-to-Sphere Transitions in the Ternary System PS 310- B-PAA 52/Dioxane/Water. Langmuir 2001, 17 (21), 67056714.

(14) Geng, Y.; Ahmed, F.; Bhasin, N.; Discher, D. E. Visualizing Worm Micelle Dynamics and Phase Transitions of a Charged Diblock Copolymer in Water. J. Phys. Chem. B 2005, 109 (9), 3772-3779.

(15) Loverde, S. M.; Ortiz, V.; Kamien, R. D.; Klein, M. L.; Discher, D. E. Curvature-Driven Molecular Demixing in the Budding and Breakup of Mixed Component Worm-Like Micelles. Soft Matter 2010, 6 (7), 1419-1425.

(16) Zhang, L.; Yu, K.; Eisenberg, A. Ion-Induced Morphological Changes in "Crew-Cut" Aggregates of Amphiphilic Block Copolymers. Science 1996, 272 (5269), 1777-1779.

(17) Cui, H.; Chen, Z.; Zhong, S.; Wooley, K. L.; Pochan, D. J. Block Copolymer Assembly via Kinetic Control. Science 2007, 317 (5838), 647-650.

(18) Meli, L.; Santiago, J. M.; Lodge, T. P. Path-Dependent Morphology and Relaxation Kinetics of Highly Amphiphilic Diblock Copolymer Micelles in Ionic Liquids. Macromolecules 2010, 43 (4), $2018-2027$.

(19) Voets, I. K.; de Keizer, A.; Stuart, M. A. C. Complex Coacervate Core Micelles. Adv. Colloid Interface Sci. 2009, 147-148 (C), 300-318.

(20) Muthukumar, M. 50th Anniversary Perspective: a Perspective on Polyelectrolyte Solutions. Macromolecules 2017, 50 (24), 9528-9560.

(21) Sing, C. E.; Perry, S. L. Recent Progress in the Science of Complex Coacervation. Soft Matter 2020, 50, 9528-9530.

(22) Krogstad, D. V.; Lynd, N. A.; Miyajima, D.; Gopez, J.; Hawker, C. J.; Kramer, E. J.; Tirrell, M. V. Structural Evolution of Polyelectrolyte Complex Core Micelles and Ordered-Phase Bulk Materials. Macromolecules 2014, 47 (22), 8026-8032.

(23) Perry, S. L.; Leon, L.; Hoffmann, K. Q.; Kade, M. J.; Priftis, D.; Black, K. A.; Wong, D.; Klein, R. A.; Pierce, C. F.; Margossian, K. O.; et al. Chirality-Selected Phase Behaviour in Ionic Polypeptide Complexes. Nat. Comm. 2015, 6, 1-8.

(24) Takahashi, R.; Narayanan, T.; Sato, T. Growth Kinetics of Polyelectrolyte Complexes Formed From Oppositely-Charged Homopolymers Studied by Time-Resolved Ultra-Small-Angle X-Ray Scattering. J. Phys. Chem. Lett. 2017, 8 (4), 737-741.

(25) Takahashi, R.; Narayanan, T.; Yusa, S.-I.; Sato, T. Kinetics of Morphological Transition Between Cylindrical and Spherical Micelles in a Mixture of Anionic-Neutral and Cationic-Neutral Block Copolymers Studied by Time-Resolved SAXS and USAXS. Macromolecules 2018, 51 (10), 3654-3662.

(26) Lund, R.; Willner, L.; Richter, D.; Lindner, P.; Narayanan, T. Kinetic Pathway of the Cylinder-to-Sphere Transition in Block Copolymer Micelles Observed in Situ by Time-Resolved Neutron and Synchrotron Scattering. ACS Macro Lett. 2013, 2 (12), 1082-1087.

(27) Ting, J. M.; Wu, H.; Herzog-Arbeitman, A.; Srivastava, S.; Tirrell, M. V. Synthesis and Assembly of Designer Styrenic Diblock Polyelectrolytes. ACS Macro Lett. 2018, 7 (6), 726-733.

(28) Wu, H.; Ting, J. M.; Weiss, T. M.; Tirrell, M. V. Interparticle Interactions in Dilute Solutions of Polyelectrolyte Complex Micelles. ACS Macro Lett. 2019, 8, 819-825.

(29) Wu, H.; Ting, J. M.; Tirrell, M. V. Mechanism of Dissociation Kinetics in Polyelectrolyte Complex Micelles. Macromolecules 2020, 53, 102-111.

(30) Smolsky, I. L.; Liu, P.; Niebuhr, M.; Ito, K.; Weiss, T. M.; Tsuruta, H. Biological Small-Angle X-Ray Scattering Facility at the 
Stanford Synchrotron Radiation Laboratory. J. Appl. Cryst 2007, 40, s453-s458.

(31) Martel, A.; Liu, P.; Weiss, T. M.; Niebuhr, M.; Tsuruta, H. An Integrated High-Throughput Data Acquisition System for Biological Solution X-Ray Scattering Studies. J. Synchrotron Rad. 2012, 19, 431-434.

(32) Franke, D.; Petoukhov, M. V.; Konarev, P. V.; Panjkovich, A.; Tuukkanen, A.; Mertens, H. D. T.; Kikhney, A. G.; Hajizadeh, N. R.; Franklin, J. M.; Jeffries, C. M.; et al. ATSAS 2.8: a Comprehensive Data Analysis Suite for Small-Angle Scattering From Macromolecular Solutions. J. Appl. Cryst. 2017, 50, 1212-1225.

(33) Ohnsorg, M. L.; Ting, J. M.; Jones, S. D.; Jung, S.; Bates, F. S.; Reineke, T. M. Tuning PNIPAm Self-Assembly and Thermoresponse: Roles of Hydrophobic End-Groups and Hydrophilic Comonomer. Polym. Chem. 2019, 10, 3469-3479.

(34) Heo, T.-Y.; Kim, I.; Chen, L.; Lee, E.; Lee, S.; Choi, S.-H. Effect of Ionic Group on the Complex Coacervate Core Micelle Structure. Polymers 2019, 11, 455.

(35) Rumyantsev, A. M.; Potemkin, I. I. Explicit Description of Complexation Between Oppositely Charged Polyelectrolytes as an Advantage of the Random Phase Approximation Over the Scaling Approach. Phys. Chem. Chem. Phys. 2017, 19, 27580-27592.

(36) Fu, J.; Schlenoff, J. B. Driving Forces for Oppositely Charged Polyion Association in Aqueous Solutions: Enthalpic, Entropic, but Not Electrostatic. J. Am. Chem. Soc. 2016, 138 (3), 980 990.

(37) Rathee, V. S.; Sidky, H.; Sikora, B. J.; Whitmer, J. K. Role of Associative Charging in the Entropy-Energy Balance of Polyelectrolyte Complexes. J. Am. Chem. Soc. 2018, 140 (45), 15319-15328.

(38) Takahashi, R.; Narayanan, T.; Sato, T. Growth Kinetics of Polyelectrolyte Complexes Formed From Oppositely-Charged Homopolymers Studied by Time-Resolved Ultra-Small-Angle X-Ray Scattering. J. Phys. Chem. Lett. 2017, 8 (4), 737-741.

(39) Zhang, J.; Chen, S.; Zhu, Z.; Liu, S. Stopped-Flow Kinetic Studies of the Formation and Disintegration of Polyion Complex Micelles in Aqueous Solution. Phys. Chem. Chem. Phys. 2014, 16, 117127.

(40) Kremer, K.; Grest, G. S. Dynamics of Entangled Linear Polymer Melts: a Molecular-Dynamics Simulation. J. Chem. Phys. 1990, $92(8), 5057-5086$.

(41) Plimpton, S. Fast Parallel Algorithms for Short-Range Molecular Dynamics. J. Comput. Phys. 1995, 117, 1-19.

(42) Bos, I.; Sprakel, J. Langevin Dynamics Simulations of the Exchange of Complex Coacervate Core Micelles: the Role of Nonelectrostatic Attraction and Polyelectrolyte Length. Macromolecules 2019, $52,8923-8931$.

(43) Perry, S. L.; Sing, C. E. 100th Anniversary of Macromolecular Science Viewpoint: Opportunities in the Physics of Sequence-Defined Polymers. ACS Macro Lett. 2020, 9, 216-225.

(44) Amann, M.; Diget, J. S.; Lyngsø, J.; Pedersen, J. S.; Narayanan, T.; Lund, R. Kinetic Pathways for Polyelectrolyte Coacervate Micelle Formation Revealed by Time-Resolved Synchrotron SAXS. Macromolecules 2019, 52, 8227-8237.

(45) Wu, H.; Ting, J. M.; Werba, O.; Meng, S.; Tirrell, M. V. Non-Equilibrium Phenomena and Kinetic Pathways in Self-Assembled Polyelectrolyte Complexes. J. Chem. Phys. 2018, 149 (16), 163330.

(46) Horn, J.; Kapelner, R.; Obermeyer, A. Macro- and Microphase Separated Protein-Polyelectrolyte Complexes: Design Parameters and Current Progress. Polymers 2019, 11 (4), 578.

(47) Acar, H.; Ting, J. M.; Srivastava, S.; LaBelle, J. L.; Tirrell, M. V. Molecular Engineering Solutions for Therapeutic Peptide Delivery. Chem. Soc. Rev. 2017, 46, 6553-6569.

(48) Van Bruggen, C.; Hexum, J. K.; Tan, Z.; Dalal, R. J.; Reineke, T. M. Nonviral Gene Delivery with Cationic Glycopolymers. Acc. Chem. Res. 2019, 52 (5), 1347-1358. 
Table of Contents Graphic

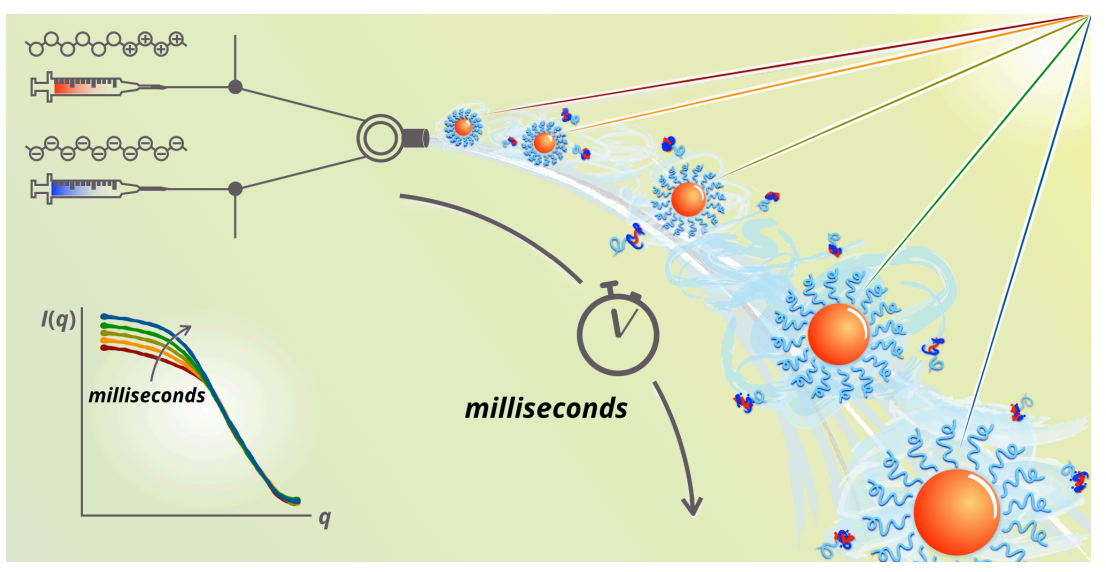

